\title{
ARTERIAL GRAFTS FOR CORONARY SURGERY: VASOSPASM AND PATENCY RATE ${ }^{\star}$
}

Guo-Wei He, MD, PhD

nterest in the use of arterial grafts in coronary artery bypass grafting (CABG) has increased significantly for the following reasons: (1) The number of patients receiving arterial grafts has risen sharply in most major cardiac surgery centers around the world; (2) the number of arterial grafts received per patient has increased; (3) more diverse arteries are used as grafts than previously; (4) more is understood about the biologic characteristics of arterial grafts; (5) clinical protocols for the use of arterial grafts are more advanced; and (6) midterm results with alternative arterial grafts are encouraging in a large number of patients.

It is widely accepted that the left internal thoracic artery (ITA) is the artery of choice for an arterial graft. Opinions differ, however, as to the second choice for an arterial graft, which frequently depends on the personal preference of the surgeon. The right ITA, the radial artery (RA), and the right gastroepiploic artery (GEA) are the candidates for second choice for arterial grafts for CABG. In general, which artery is chosen depends on the surgeon's views about the quality of the graft, that is, its length and diameter, whether the artery can be used as a pedicle graft, the technical difficulties, the incidence of vasospasm, and the possible superior longterm patency. Usually, pedicle grafts are considered to be superior to free grafts because the anatomic structure of the pedicle artery is thought to be more intact (the nerve and the blood supply to the vaso vasorum are better preserved in the pedicle graft). On the other hand, for arterial grafts such as the GEA, the pattern of direct flow from the ascending aorta in the free graft is better than that from the abdominal aorta (in the in situ pedicle graft). Therefore, although the ITA is better used as a pedicle graft than as a free graft, the GEA, when used as a free graft, has some advantages with regard to the flow pattern.

\footnotetext{
*For related article, see p. 520.

From the Division of Cardiothoracic Surgery, Department of Surgery, The Chinese University of Hong Kong, Hong Kong SAR, China.

J Thorac Cardiovasc Surg 2001;121:431-3

Copyright $\odot 2001$ by The American Association for Thoracic Surgery

$0022-5223 / 2001 \$ 35.00+0 \quad \mathbf{1 2 / 1 / 1 1 3 5 9 3}$

doi: $10.1067 / \mathrm{mtc} .2001 .113593$
}

Because the most important advantage for arterial grafts compared with vein grafts is their superior longterm patency, any alteration of the technique used for all kinds of arterial grafts should also take long-term patency into account. The long-term patency of arterial grafts is related to many factors:

1. Biologic characteristics of the arterial grafts, including (a) graft diameter, (b) the thickness of the wall and of the intimal hyperplasia, (c) the anatomic structure of the graft, (d) the usable length of the graft and whether it can be used as a pedicle graft, (e) the incidence of vasospasm, and (f) the incidence of atherosclerosis and occlusion rate both in situ and in the grafts.

2. General risk factors for coronary artery disease such as diabetes mellitus, hypertension, and hypercholesterolemia.

3. Anatomy and pathologic status of the target coronary artery, and disease status of the native coronary artery, such as the degree of stenosis, the quality of the wall, the flow capacity of the runoff, and the size of the vessel.

4. Vessel match between the graft and the target coronary artery.

5. The flow pattern through the graft, which may also be related to the patency.

6. Technical considerations, which depend primarily on the operative technique of the surgeon.

7. Function of endothelium and smooth muscle: in particular, preservation of endothelial function during the preparation of the graft is crucial to long-term patency.

8. Pharmacologic therapy: (a) perioperative antispastic protocol for arterial grafts and (b) antiplatelet therapy (aspirin) for long-term patency.

The surgeon can control some of the preceding factors during surgery by doing the following:

1. Choosing an adequate graft.

2. Using a pedicle graft whenever possible.

3. Improving operative skills to reduce the risk of graft occlusion by technical factors.

4. Using an adequate antispastic protocol during graft preparation to reduce damage to the endothelium and the smooth muscle of the graft.

Arterial grafts are small to mid-sized conduit arteries. A major concern of arterial grafting is vasospasm in the graft perioperatively. Because of the lack of data com- 
paring these grafts, we have no direct evidence that the incidence of vasospasm in the free graft is higher or lower than that in the pedicle graft.

Despite numerous studies on the causes of vasospasm in arterial grafts in particular and in all kinds of vessels in general, little is known about how vasospasm develops. The correlation between vasospasm and the reactivity of a vessel to vasoconstrictors is also unclear. However, it is presumed that vasospasm is an extreme form of vasoconstriction that may be the response of a vessel to many stimuli (spasmogens). These stimuli may be physical (such as mechanical stimulation or temperature change) or pharmacologic (such as nerve stimulators or vasoconstrictor substances).

Important vasoconstrictor substances, which may act as spasmogens for blood vessels, ${ }^{1}$ are (1) endotheliumderived contracting factors such as endothelin, (2) prostanoids such as thromboxane $\mathrm{A}_{2}$ and prostaglandin $\mathrm{F}_{2 \alpha}$, (3) circulating sympathomimetic substances $(\alpha-$ adrenoceptor agonists) such as norepinephrine and synthetic $\alpha_{1}$-adrenoceptor agonists (methoxamine or phenylephrine), (4) platelet-derived contracting substances such as 5-hydroxytryptamine and thromboxane $\mathrm{A}_{2}$, (5) substances released from mast cells and basophils such as histamine, (6) muscarinic receptor agonists such as acetylcholine, (7) renin-angiotensin system-related substances such as angiotensin II, (8) contracting vasopressin substances, and (9) the depolarizing agent potassium ion.

The contractility of arterial grafts in response to the preceding vasoconstrictors has been studied extensively. Although all arterial grafts react to these vasoconstrictors, some arteries react to vasoconstrictors more strongly than others do. This is best reflected by the fact that the GEA reacts more strongly to the vasoconstrictors $\mathrm{K}^{+}$, thromboxane $\mathrm{A}_{2}$, endothelin-1, and norepinephrine than do other arteries. ${ }^{2}$ Another example is that the RA reacts more strongly to receptor-mediated contractions than does the ITA. ${ }^{3}$ Clinically, although vasospasm may develop in all arterial grafts, it develops more frequently in the GEA and RA than in the ITA and inferior epigastric artery. Postoperative vasospasm and occlusion account for the early abandonment of the RA and is considered a possible cause for abandoning the GEA in some cardiac surgical centers. These studies led us to classify arterial grafts as type I (somatic arteries), type II (splanchnic arteries), and type III (limb arteries)., 2,4 Types II and III are more spastic. Vasospasm is seen frequently in the GEA, as a type II artery, during harvesting.

Further, vasospasm in arterial grafts is clearly related to endothelial function. Our most recent studies show differences in the release of nitric oxide and endotheli- um-derived hyperpolarizing factor, 5,6 both between the ITA and the saphenous vein and between the RA and ITA. Development of vasospasm in the arterial graft is clearly related to endothelial function. When endothelial function is damaged, such as occurs with inadequate preparation of the graft, the graft has a tendency to go into vasospasm.

In the article by Matsuura and colleagues in this issue of the Journal, the authors developed a new method (transplantation of an en bloc vascular system) for overcoming vasospasm in the GEA. This method is characterized by two major points-use of a free GEA graft and establishment of venous drainage.

The authors hypothesized that the vasospasm seen in the GEA is related to disconnected venous return of the GEA and the attached omental tissue because they observed swelling of the free GEA graft. They therefore developed a new technique to anastomose the venous return to the right atrium to establish the circulation of the vaso vasorum. Preliminary results indicate that this method seems to reduce swelling of the tissue. In the 33 patients observed, no vasospasm occurred postoperatively. The authors concluded that free GEA grafting and the venous drainage technique prevent graft spasm, leading to an improved patency rate.

Before the method can be widely accepted, however, several points should be emphasized:

First, as mentioned previously, the exact cause of vasospasm is unknown. There are no data to support the authors' hypothesis that the main cause of free graft spasm is graft damage caused by interruption of venous drainage from the graft. Also, the exact cause of vasospasm is still unknown. The difference between the incidence of vasospasm in the free GEA and that in the in situ GEA is not established. Similarly, the incidence of vasospasm in the free right ITA graft is not higher than that in the pedicled right ITA.

Second, the relationship between vasospasm in arterial grafts and venous return is unclear. The authors' hypothesis is therefore premature. Again, in right ITA grafting, there is usually no need for direct venous drainage to the right atrium from the graft and vasospasm is not seen more frequently in the right ITA. The authors also noticed that the need to establish venous return is unique for GEA, if there is such a need.

Other aspects should also be carefully considered. First, those who developed GEA grafting and who have performed the procedure in a large number of patients, such as Suma, ${ }^{7} \mathrm{Pym},{ }^{8}$ and their associates, have not emphasized the need to establish venous return for GEA. Others ${ }^{9}$ also reported an $84.5 \%$ patency rate at 10 months and a $97 \%$ early patency rate for GEA with- 
out performing the venous anastomosis for venous return. The excellent results from these experiences may lead one to question the necessity of establishing venous return in the GEA graft. Second, it may be unnecessary to establish venous return surgically in skeletonized or semiskeletonized GEA grafts, as is the case in other arterial grafts. Third, histologic studies ${ }^{10}$ have shown that arterial grafts can be nourished through the lumen, thereby indicating the possible presence of a venous return mechanism directly to the lumen that is presumably enough if there is no redundant tissue around the graft.

To support their hypothesis, the authors should have a control group in which the free GEA graft is used without venous drainage to the right atrium. The incidence of vasospasm and the long-term patency should be compared in these two groups. Without such a control, it would be difficult to conclude that the establishment of the venous return would reduce the incidence of vasospasm in the free GEA graft. Further, anatomic studies may be necessary to investigate the possible venous return mechanism through the wall of the GEA directly to the lumen.

Received for publication Nov 29, 2000; accepted for publication Dec 1, 2000.

Address for reprints: Professor Guo-Wei He, Department of Surgery, The Chinese University of Hong Kong, Block B, 5A, Prince of Wales Hospital, Shatin, NT, Hong Kong SAR, China (E-mail: gwhe@cuhk.edu.hk).

\section{REFERENCES}

1. He G-W, Yang C-Q, Starr A. An overview of the nature of vasoconstriction in arterial grafts for coronary surgery. Ann Thorac Surg 1995;59:676-83.

2. He G-W, Yang C-Q. Comparison among arterial grafts and coronary artery: an attempt at functional classification. $\mathrm{J}$ Thorac Cardiovasc Surg 1995;109:707-15.

3. He G-W, Yang C-Q. Radial artery has higher receptor-selective contractility but similar endothelium function compared to mammary artery. Ann Thorac Surg 1997;63:1346-52.

4. He G-W. Arterial grafts for coronary artery bypass: biological characteristics, functional classification, and clinical choice (current review). Ann Thorac Surg 1999;67:277-84.

5. He G-W, Liu Z-G. Comparison of nitric oxide release and endothelium-derived hyperpolarizing factor-mediated hyperpolarization between human internal thoracic and radial artery [abstract]. Circulation 2000;102(Suppl):II-402 .

6. Liu Z-G, Ge Z-D, He G-W. Difference in hyperpolarization between human internal mammary artery and saphenous vein. Circulation 2000;102(Suppl):III-296-301.

7. Suma H, Wanibuchi Y, Terada Y, Fukuda S, Takayama T, Furuta $S$. The right epigastric artery graft: clinical and angiographic midterm results in 200 patients. J Thorac Cardiovasc Surg 1993; 105:615-23

8. Pym J, Brown P, Pearson M, Parker J. Right epigastric-to-coronary artery bypass: the first decade of use. Circulation 1995;92(Suppl):II-45-9.

9. Grandjean JG, Boonstra PW, den Heyer P, Ebels T. Arterial revascularization with the right epigastric artery and internal mammary arteries in 300 patients. J Thorac Cardiovasc Surg 1994;107:1309-16.

10. van Son JAM, Smedts F, Vincent JG, van Lier HJJ, Kubat K. Comparative anatomic studies of various arterial conduits for myocardial revascularization. J Thorac Cardiovasc Surg 1990;99:703-7.

\section{Notice of correction}

In the January 2001 issue of the Journal, in the article by Rodriguez and associates titled "Effect of Trendelenburg Head Position During Cardiac Deairing on Cerebral Microemboli in Children: A Randomized Controlled Trial" (2001;121:3-9), an error was made. On page 3 the name of one author, Ba' Pham, MSc, was omitted. The correct listing of authors is as follows:

Rosendo A. Rodriguez, MD, $\mathrm{PhD}^{\mathrm{a}}$

Garry Cornel, MB, BS

Nihal A. Weerasena, MB, BS

Ba' Pham, MSc ${ }^{\mathrm{b}}$

William M. Splinter, MD $^{\mathrm{c}}$ 